\title{
Genetic variation in the functional ENG allele inherited from the non-affected parent associates with presence of pulmonary arteriovenous malformation in hereditary hemorrhagic telangiectasia 1 (HHT1) and may influence expression of PTPN14
}

\author{
Tom G. W. Letteboer ${ }^{1,2 \neq}$, Michael Benzinou ${ }^{1 \neq}$, Christopher B. Merrick ${ }^{1,3 \neq}$, David A. Quigley ${ }^{1}$, \\ Kechen Zhau ${ }^{1+}$, II-Jin Kim ${ }^{4}$, Minh D. To ${ }^{1,4}$, David M. Jablons ${ }^{4}$, Johannes K. P. van Amstel ${ }^{2}$, \\ Cornelius J. J. Westermann ${ }^{5}$, Sophie Giraud ${ }^{6}$, Sophie Dupuis-Girod ${ }^{6}$, Gaetan Lesca $^{6}$, \\ Jonathan H. Berg ${ }^{3}$, Allan Balmain ${ }^{1,78}$ and Rosemary J. Akhurst ${ }^{1,8,9 *}$ \\ 1 Helen Diller Family Comprehensive Cancer Center, University of California, San Francisco, San Francisco, CA, USA \\ ${ }^{2}$ Department of Medical Genetics, University Medical Centre Utrecht, Utrecht, Netherlands \\ ${ }^{3}$ Department of Clinical Genetics, University of Dundee, Dundee, UK \\ ${ }^{4}$ Department of Surgery, University of California, San Francisco, San Francisco, CA, USA \\ ${ }^{5}$ Department of Pulmonology, St. Antonius Hospital, Nieuwegein, Netherlands \\ ${ }^{6}$ Department of Medical Genetics, Lyon University Hospital, Lyon, France \\ 7 Department of Biochemistry and Biophysics, University of California, San Francisco, San Francisco, CA, USA \\ ${ }^{8}$ Institute of Human Genetics, University of California, San Francisco, San Francisco, CA, USA \\ ${ }^{9}$ Department of Anatomy, University of California, San Francisco, San Francisco, CA, USA
}

\section{Edited by:}

Michelle Letarte, University of

Toronto and Hospital for Sick

Children, Canada

Reviewed by:

Douglas A. Marchuk, Duke

University School of Medicine, USA

Claire L. Shovlin, Imperial College

London, UK

*Correspondence:

Rosemary J. Akhurst, Helen Diller

Family Comprehensive Cancer

Center, University of California at

San Francisco, Helen Diller Family

Cancer Research Building, 1450, 3rd

Street, San Francisco, CA

94158-9001, USA

e-mail: rakhurst@cc.ucsf.edu

${ }^{\dagger}$ Present address:

Kechen Zhau, Department of

Preventive Medicine, University of

Southern California, Los Angeles,

CA, USA

FThese authors have contributed equally to this work.
HHT shows clinical variability within and between families. Organ site and prevalence of arteriovenous malformations (AVMs) depend on the HHT causative gene and on environmental and genetic modifiers. We tested whether variation in the functional ENG allele, inherited from the unaffected parent, alters risk for pulmonary AVM in HHT1 mutation carriers who are ENG haploinsufficient. Genetic association was found between rs 10987746 of the wild type ENG allele and presence of pulmonary AVM [relative risk $=1.3$ (1.0018-1.7424)]. The rs10987746-C at-risk allele associated with lower expression of ENG RNA in a panel of human lymphoblastoid cell lines $(P=0.004)$. Moreover, in angiogenically active human lung adenocarcinoma tissue, but not in uninvolved quiescent lung, rs 10987746-C was correlated with expression of PTPN14 $(P=0.004)$, another modifier of HHT. Quantitative TAQMAN expression analysis in a panel of normal lung tissues from 69 genetically heterogeneous inter-specific backcross mice, demonstrated strong correlation between expression levels of Eng, Acvrl1, and Ptpn14 ( $r 2=0.75-0.9$, $\left.P<1 \times 10^{-12}\right)$, further suggesting a direct or indirect interaction between these three genes in lung in vivo. Our data indicate that genetic variation within the single functional ENG gene influences quantitative and/or qualitative differences in ENG expression that contribute to risk of pulmonary AVM in HHT1, and provide correlative support for PTPN14 involvement in endoglin/ALK1 lung biology in vivo. PTPN14 has been shown to be a negative regulator of $\mathrm{Yap} / \mathrm{Taz}$ signaling, which is implicated in mechanotransduction, providing a possible molecular link between endoglin/ALK1 signaling and mechanical stress. EMILIN2, which showed suggestive genetic association with pulmonary AVM, is also reported to interact with Taz in angiogenesis. Elucidation of the molecular mechanisms regulating these interactions in endothelial cells may ultimately provide more rational choices for HHT therapy.

Keywords: endoglin, ACVRL1, PTPN14, EMILIN2, lung, arteriovenous malformations

\section{INTRODUCTION}

Hereditary Hemorrhagic Telangiectasia (HHT) is an autosomal dominant disorder resulting from loss of function mutations predominantly in ENG (HHT type 1) or ACVRL1 aka ALK1 (HHT type 2) (Shovlin, 2010; Faughnan et al., 2011). These genes encode cell surface receptors which are components of the TGF- $\beta /$ BMP signal transduction pathways active predominantly in endothelial cells. Endoglin $(E N G)$ is a trans-membrane glycoprotein similar 
to the TGF- $\beta$ type III receptor and ALK1 (ACVRL1) is a transmembrane serine threonine kinase TGF- $\beta / \mathrm{BMP}$ type I receptor (Lebrin et al., 2004; Lopez-Novoa and Bernabeu, 2010; Pardali et al., 2010; Cunha and Pietras, 2011). Affected HHT patients typically present during adolescence with muco-cutaneous telangiectases and frequent severe episodes of epistaxis (Shovlin, 2010; Faughnan et al., 2011). Telangiectases result from abnormal vascular remodeling, leading to capillary breakdown and consequent shunting of arterioles directly into venules with no intervening capillary bed (Guttmacher et al., 1995). Blood vessel walls of lesions are dilated and weak, leading to frequent hemorrhage. Epistaxis and bleeding from gastrointestinal tract telangiectases can result in severe anemia and the need for frequent blood transfusions. HHT is highly penetrant, but with age dependent expression of different clinical features (Shovlin, 2010; Faughnan et al., 2011).

In addition to telangiectases, approximately half of HHT patients develop more serious complications, namely visceral AVMs that can occur anywhere but are mostly found in lung, brain, and liver. AVMs also involve shunting of blood directly from artery to vein. Large AVMs lead to local hypoxia and are prone to hemorrhage. Brain AVMs are often asymptomatic, but can cause seizures or hemorrhage. Pulmonary AVMs are at high risk of thromboembolism, resulting in thrombotic stroke or cerebral abscess. One of the features of HHT is a large range in clinical severity and prevalence of AVMs (Letteboer et al., 2006; Lesca et al., 2007; Sabba et al., 2007). Some phenotypic variability can be accounted for by genetic heterogeneity, for example lung AVMs are more common in HHT1, whereas hepatic AVMs are more prevalent in HHT2 (Letteboer et al., 2006; Lesca et al., 2007; Sabba et al., 2007). Nevertheless, the large intra-familial range in clinical severity suggests that local environmental stresses, such as wounding and/or inflammation (van Laake et al., 2006; Park et al., 2009; Garrido-Martin et al., 2014) and/or germline genetic modifiers (Benzinou et al., 2012; Kawasaki et al., 2014), may contribute to precipitation of telangiectases or AVMs. Localized somatic mutation (Akers et al., 2009; McDonald et al., 2014) might also contribute to the risk of clinical progression, although this has not been definitively tested. Identification of diseasemodifying germline genetic variants may provide a deeper molecular understanding of the process of AVM formation that might be harnessed for therapeutic design purposes.

In order to uncover possible molecular mechanisms that contribute to pulmonary AVMs, we previously undertook a screen for genetic loci that associate with pulmonary AVM prevalence within the HHT population (Benzinou et al., 2012). As TGF- $\beta 1$ is known to bind both endoglin and ALK1 and attenuate or activate signaling downstream of ALK1, we hypothesized that genetic modifiers of TGF- $\beta 1$ ( Tgfbm's) may influence the vascular outcome of HHT. We therefore screened genes within human loci syntenic to mouse $T g f b m 2$ and $T g f b m 3$, both suppressors of a lethal prenatal vascular dysplasia observed in some $T g f b 1-/-$ mice (Benzinou et al., 2012; Kawasaki et al., 2014). We also screened for genetic association within 72 additional human genes, genomewide, that encode components of the TGF- $\beta$ or BMP signaling pathways, or that had been implicated in regulating or being regulated by TGF- $\beta$ or BMP (Benzinou et al., 2012). While we found only one gene, PTPN14, encoding protein tyrosine phosphatase, non-receptor type 14 (PTPN14), that fit our stringent genetic criteria for being a modifier of pulmonary AVM incidence in HHT (Benzinou et al., 2012), the current study focuses on weaker but still instructive genetic associations. We also provide further correlative evidence that PTPN14 interacts with ENG in the lung in vivo.

\section{METHODS \\ HHT HUMAN SUBJECTS}

Study protocols were approved by local ethics committees at all institutions, and informed consent was obtained from each subject before participation in the studies. Initially, 649 Dutch Caucasians and 72 Dutch Antillean Blacks were genotyped. These were selected from a panel consisting of probands and family members from a total of 137 different families screened for HHT. All manifestations of HHT were recorded for both probands and family members. The clinical diagnosis of HHT was established according to the Curaçao criteria (Faughnan et al., 2011), which are clinical features of epistaxis, multiple telangiectases, visceral AVMs and a first-degree relative with HHT. A diagnosis of HHT is considered definitive if three criteria are present, possible if two criteria are present and unlikely if fewer than two criteria are present. Mutation carrier status was confirmed by molecular analysis. Most probands and family members were screened for visceral manifestations at St Antonius Hospital, which specializes in the diagnosis and treatment of HHT. Screening for the presence of pulmonary AVMs was performed routinely by chest radiography and by measuring partial oxygen tension in arterial blood and, if abnormal, followed by the $100 \%$ oxygen right-to-left shunt test. Based on this analysis, patients with suspected pulmonary AVMs were offered conventional angiography, digital subtraction angiography of the pulmonary arteries or computed tomography (CT) of the chest. In some patients, pulmonary AVMs were diagnosed using high-resolution CT and/or contrast trans-thoracic echocardiography.

HHT clinical diagnosis in the French cohort was also established according to the Curaçao criteria. Mutation carrier status was confirmed by molecular diagnosis (heteroduplex analysis or denaturing high performance liquid chromatography and sequencing). The 222 French patients included in the present study all carried a mutation in either ENG $(n=76)$ or ACVRL1 ( $n=146)$, and $43 \%$ of them had pulmonary AVMs $(74 \%$ for HHT1 patients and $27 \%$ for HHT2 patients). Familial structures included 111 singletons, 40 duos, 2 trios, 5 quartets, and 1 family with 5 individuals. The diagnosis of pulmonary involvement was made either in patients presenting symptoms (for example, dyspnoea and cyanosis) or complications (mainly brain abscess), or in asymptomatic HHT patients who underwent screening using contrast trans-thoracic echocardiography, chest radiograph and/or oxygen shunt test as described. Screening for pulmonary AVMs was also recommended to asymptomatic patients and accepted by a majority of them (Lesca et al., 2007). The "no-pulmonary AVM" cohort should thus be considered as either negative for pulmonary AVMs or having only small clinically-insignificant pulmonary AVMs at the time of assessment. 


\section{LYMPHOBLASTOID CELL LINES}

Affymetrix gene expression data for 61 human lymphoblastoid cell lines derived from blood samples from Utah residents of Northern and Western European Ancestry from the CEPH collection (CEU) (Cheung et al., 2005), were downloaded from Gene Expression Omnibus (GEO), and matching genotype data for $r$ s10987746 was downloaded from the International HapMap Project website. Expression levels of ENG and PTPN14 were compared by $r s 10987746$ genotype. Since the $r s 10987746-C / C$ genotype was unusually underrepresented in this panel $(n=7)$, and showed no statistically significant difference in expression from the $r$ s10987746-T/C genotype, these two genotypes were pooled and their combined expression levels compared to that of the $r$ s10987746-T/T genotype. P-values (ANOVA) were calculated using Tukey's HSD in R Development Core Team (2011).

\section{HUMAN LUNG CANCER SUBJECTS}

All samples were obtained from patients with informed consent under the IRB approval (ID: 10-03352) granted by the Committee on Human Research at the University of California, San Francisco (UCSF). Paired RNA and DNA samples from human primary lung adenocarcinomas and uninvolved "normal" lung tissue were obtained from patients undergoing tumor debulking surgery at UCSF. Each tumor sample consisted of at least 50\% tumor cells.

\section{MICROARRAY EXPRESSION ANALYSIS OF PAIRED NORMAL AND ADENOCARCINOMA LUNG TISSUE FROM LUNG CANCER PATIENTS}

RNA was extracted with Trizol followed by DNAse I treatment and purification (RNeasy RNA purification kit, Qiagen). The purified RNA was quantified with Nanodrop and QC checked by Agilent Bioanalyzer. Gene expression was measured with the Illumina Human Whole Genome 62.0 array (Illumina Inc., San Diego, CA, USA). Sample preparations, hybridization and scanning were performed according to manufacturer's instructions. Briefly, $200 \mathrm{ng}$ of total RNA was prepared to make cRNA by using Illumina TotalPrep RNA Amplification Kit (Ambion). Firststrand complementary DNA was generated with T7 oligo (dT) primer and ArrayScript and then second cDNA was also made with DNA polymerase. Biotin-NTP with T7 enzyme mixes was used to make biotinylated cRNAs. The labeled cRNAs were purified, quantified and checked again using the Bioanalyzer. The labeled cRNA target was used for hybridization and scanning according to Illumina Human Whole Genome 62.0 array protocol. Probes with present/absent call $\geq 0.05$ assessed by Illumina Beadstudio software, were marked absent. Raw microarray data were quantile normalized and $\log 2$-transformed. Statistical analysis was performed with $\mathrm{R}$ version 2.13 (R Development Core Team, 2011).

\section{HUMAN SNP SELECTION}

Gene-centric tag-SNPs were chosen on the basis of being nonsynonymous coding polymorphisms, potential splice site variants or inferred to disrupt important gene regulatory sites (that is, microRNAs and transcription factor binding sites, and so on) using the UCSC genome server. We further looked for SNPs located in highly conserved regions in the $3^{\prime}$ UTR. This first set of SNPs was complemented by tag-SNPs using a tag approach with the HapMap phase II database (http://www.hapmap.org) by "force including" the first set of variants using the Haploview Tagger and by searching for additional tag-SNPs. Selected SNPs were estimated to give $\sim 85 \%$ coverage of any chosen gene.

\section{HUMAN GENOTYPING AND QUALITY CONTROL}

We previously screened genetic variants for association with the presence of pulmonary AVM in HHT patients from 137 Dutch HHT families. The first screen included 649 Dutch individuals of northern European descent and 72 Afro-Caribbean individuals from the Dutch Antilles. In addition to screening SNPs within TGFBM2 (Benzinou et al., 2012) and TGFBM3 (Kawasaki et al., 2014), we screened tag-SNPs $(n=443)$ that covered 72 "candidate genes" selected on the basis of their involvement in TGF- $\beta$ or BMP signaling and/or their responses to TGF- $\beta$. We used a modification of the transmission disequilibrium test (TDT), namely Gamete Competition (GC) (Lange et al., 2001, 2005) to screen for genetic association with the presence $v s$. absence of pulmonary AVM in HHT mutation carriers. GC was selected over TDT because of the large, multigenerational structure of families within this cohort. In addition to the previously published genetic associations (Benzinou et al., 2012; Kawasaki et al., 2014) 17 of the 443 SNPs tagging candidate genes showed nominal evidence of over-transmission to pulmonary AVM + in Dutch HHT patients $(P<0.05$, GC test), and were genotyped in an additional 108 northern European Dutch individuals (Extension study). Genotyping of the first Dutch cohort was performed using 750-ng labeled genomic DNA hybridized to a custom Illumina chip. Genotyping for the Dutch extension and French replication studies was performed using Sequenom MALDI-TOF mass spectrometry. No significant difference in call rates between cases and controls was seen. Samples successfully genotyped in $<95 \%$ of markers were excluded from analysis. Markers were excluded if they deviated significantly from Hardy-Weinberg equilibrium $(P<0.05$, Hardy-Weinberg $)$ or if they had a call rate $<95 \%$ in the entire cohort.

\section{EXTRACTION OF RNA FROM A MUS SPRETUS $\times$ MUS MUSCULUS F1 BACKCROSS}

All animal experiments were approved a priori by the UCSF IACUC. Backcross mice were generated by crossing inbred male SPRET/Ei with inbred female FVB/N mice (Jackson Laboratory). Female F1 hybrids were then mated to male FVB/N mice. Lungs from eight-week-old mice were snap-frozen, and RNA was isolated using TRIzol (Invitrogen) according to the manufacturer's instructions. Residual contaminating genomic DNA was removed by DNase treatment (Ambion).

\section{qRT-PCR TAQMAN ANALYSIS}

PCR was conducted in triplicate with $20 \mu \mathrm{L}$ reaction volumes of 1X Taqman buffer (1X Applied Biosystems PCR buffer, $20 \%$ glycerol, $2.5 \%$ gelatin, $60 \mathrm{nM}$ Rox as a passive reference), $5.5 \mathrm{mM} \mathrm{MgCl} 2,0.5 \mathrm{mM}$ each primer, $0.2 \mu \mathrm{M}$ each deoxynucleotide triphosphate (dNTP), $200 \mathrm{nM}$ probe, and $0.025 \mathrm{unit} / \mu \mathrm{L}$ AmpliTaq Gold (Applied Biosystems) with 5 ng cDNA. A large master mix of the above-mentioned components (minus the primers, probe, and CDNA) was made for each experiment and 
aliquoted into individual tubes, one for each cDNA sample. cDNA was then added to the aliquoted master mix. The master mix with cDNA was aliquoted into a 384-well plate. The primers and probes were mixed together and added to the master mix and cDNA in the 384-well plate. PCR was conducted on the ABI 7900HT (Applied Biosystems) using the following cycle parameters: 1 cycle of $95^{\circ}$ for $10 \mathrm{~min}$ and 40 cycles of $95^{\circ}$ for $15 \mathrm{~s}, 60^{\circ}$ for $1 \mathrm{~min}$. SDS Analysis (version 2.3) supplied with ABI 7900HT, was used to determine the $C t$ values of each reaction.

$C t$ values were determined for three test and three reference reactions in each sample, averaged, and subtracted to obtain the $\Delta \mathrm{Ct}[\Delta \mathrm{Ct}=\mathrm{Ct}$ (test locus) $-\mathrm{Ct}$ (control locus)]. PCR efficiencies were measured for all custom assays and were $=90 \%$. Therefore, relative fold difference was calculated for each primer/probe combination as $2^{-\Delta \mathrm{Ct}} \times 100$.

Primer probe combinations were either generated in house (mouse Gus and rat Gapdh):

Gus

Forward: CTCATCTGGAATTTCGCCGA

Reverse: GGCGAGTGAAGATCCCCTTC

Probe: CGAACCAGTCACCGCTGAGAGTAATCG

GapDH

Forward: TGCACCACCAACTGCTTAG

Reverse: GGATGCAGGGATGATGTTC

Probe: CAGAAGACTGTGGATGGCCCCTC

or purchased as proprietary reagents from ABI-Life Technologies Inc:

Eng, mCG18573: Assay ID: Mm00468256_m1

Acvrl1, mCG16831: Assay ID: Mm00437432_m1

Ptpn14, mCG19670: Assay ID: Mm00501215_m1

\section{RESULTS}

\section{GENETIC VARIATION IN THE FUNCTIONAL ENG ALLELE ASSOCIATES WITH PULMONARY AVMS IN HHT1}

As previously reported, a candidate screen for genetic association to pulmonary AVMs in HHT patients showed that PTPN14 within the TGFBM2 locus showed the strongest genetic association to pulmonary AVM incidence in both HHT1 and HHT2 mutation carriers (Benzinou et al., 2012). However, as presented here, additional SNPs within several candidate genes showed suggestive association within the Dutch HHT population (see Supplementary Table S1). Interestingly, in the Dutch HHT cohort, primarily within HHT1, we found evidence of suggestive genetic association between the prevalence of pulmonary AVMs in three out of seven tagSNPs spanning the HHT1 causative gene, ENG $\left(P=5.4 \times 10^{-3}\right.$ for the intronic SNP $r$ s 10987746 in HHT1; GC test, Supplementary Table S2), and a similar observation was made within the French HHT1 population ( $r s 1887266, P=$ 0.005; $r s 10987746 ; P=0.087$; Supplementary Table S3). Our initial analysis did not differentiate between the two alleles of the mutated causative gene. We therefore considered that this observation might be artifactual, driven by linkage disequilibrium between the test SNP and the causative ENG mutation. Since most causative HHT mutations are loss of function, we surmised that genetic variants within the mutated gene would be functionally inconsequential whereas genetic variation within the wild type allele, inherited from the unaffected parent, may influence the clinical features of HHT. In order to interrogate this further, we limited our analysis to those Dutch HHT families for whom the genetic phase of the causative mutation could be determined. Pedigree analysis permitted us to elucidate the genetic phase of ENG rs 10987746 with respect to the causative vs. wild type ENG alleles in 266 HHT1 cases from 80 families. We tested for genetic association between $r s 10987746$ within the wild type ENG allele and the prevalence of pulmonary AVMs in Dutch HHT1, and confirmed the notion that genetic variation within the functional $E N G$ allele inherited from the non-affected parent associates with presence of pulmonary AVM in HHT1 ( $r$ 10987746-C relative risk = $1.3(1.0018-1.7424)$ (Table 1).

\section{POSSIBLE GENETIC ASSOCIATION BETWEEN ACVRL1 AND PULMONARY AVMs IN HHT2}

A similar effect to that seen with $E N G$ may occur for the wild type $A C V R L 1$ allele in HHT2. Indeed, four out of six tagSNPs that span the two adjacent loci $A C V R L 1$ and $A C V R 1 B$ showed genetic association with pulmonary AVMs in Dutch HHT2 patients $(P<$ 0.05; GC test, Supplementary Table S2). However, there were insufficient HHT2 specimens within the Dutch HHT population to undertake genetic phase analysis for $A C V R L 1$, and extensive pedigree information was not available for the French cohort, preempting an analysis of the effect of variation in the functional $A C V R L 1$ allele on pulmonary AVM incidence.

\section{SUGGESTIVE GENETIC ASSOCIATION BETWEEN EMILIN2 VARIANTS AND PULMONARY AVMs IN HHT PATIENTS}

SNPs within several other candidate genes showed suggestive association $(p<0.05)$ within the Dutch HHT population (see Supplementary Table S1). Of three EMILIN2 SNPs tested, one showed significant $(P=0.023)$ and one showed a trend toward $(P=0.09)$ association with the presence of pulmonary AVMs in the French HHT validation cohort (Supplementary Table S3). SNPs in other candidate genes with association in the Dutch screen did not validate in the French HHT cohort (not shown). The EMILIN2 association with pulmonary AVMs was weak, but was found in HHT1 only (not HHT2) in both the French and Dutch cohorts. It could represent a false positive due to issues with multiple testing, thus functional validation remains to be undertaken.

Table 1 | Genetic variation in the wild type ENG allele, inherited from the unaffected parent, associates with presence of pulmonary AVMs in $\mathrm{HHT} 1$ patients.

\begin{tabular}{lcc}
\hline $\begin{array}{l}\text { rs 10987746 genotype of } \\
\text { wild type ENG allele }\end{array}$ & $\begin{array}{c}\text { HHT1 patients } \\
\text { with pulmonary AVM }\end{array}$ & $\begin{array}{c}\text { HHT1 patients } \\
\text { no pulmonary AVM }\end{array}$ \\
\hline $\mathrm{T}$ & $63(44.4 \%)$ & $79(55.6 \%)$ \\
$\mathrm{C}$ & $70(56.5 \%)$ & $54(43.5 \%)$ \\
\hline
\end{tabular}

Relative risk of pulmonary AVMs $=1.3(95 \%$ confidence interval $=1.002-1.742)$ $P=0.018$. 
THE PULMONARY AVM-PROTECTIVE ENG ALLELE ASSOCIATES WITH HIGHER ENG EXPRESSION IN HUMAN LB CELL LINES AND TRENDS

\section{WITH HIGHER PTPN14 EXPRESSION}

$r s 1887266$ is a $5^{\prime}$ flanking SNP and $r s 10987746$ is located within intron 12 near the $3^{\prime}$ end of the gene. These might associate with quantitative differences in $E N G$ transcript levels and/or with differential ENG splicing resulting in qualitative differences in endoglin protein. We undertook eQTL analysis on publically available genome wide SNP and microarray expression data from 61 CEPH lymphoblastoid (LB) cell lines for which $r s 10987746$ had been genotyped, to investigate whether this SNP might show correlation to expression of ENG message levels. ENG mRNA was expressed at low levels in these cell lines (Figure 1A) and the frequency of $r$ s $10987746-C$ homozygosity was exceedingly low $(n=$ 7), precluding a statistically robust comparative analysis of $E N G$ transcript levels in $r s 10987746-C C$ genotype vs. rs10987746-TC or $r s 10987746-T T$ genotypes. However, using a dominant model of action for the pulmonary AVM-at-risk allele, $r s 10987746-C$, we found significant association with lower ENG expression levels $\left(P=4 \times 10^{-3}, A N O V A\right)$ (Figure 1A).

Since genetic variation within PTPN14 is also a genetic risk factor for pulmonary AVMs in HHT1 and HHT2 (Benzinou et al., 2012), we examined association between ENG-rs10987746-C and expression of PTPN14 transcript levels in the human LB dataset. PTPN14 expression in the CEPH LB cell panel was lower than that of $E N G$ and there was a trend toward association between rs10987746-C and lower PTPN14 gene expression, although this did not reach significance $(P=0.08, A N O V A$; Figure 1B).

\section{IN ANGIOGENICALLY ACTIVE HUMAN LUNG TUMOR TISSUE, BUT NOT IN NORMAL LUNG, THE PULMONARY AVM AT-RISK ENG SNP ASSOCIATES WITH HIGHER PTPN14 EXPRESSION}

Since the target organ of interest in this study is lung, we undertook eQTL analysis in a panel of human lung tissues. We genotyped rs10987746 in genomic DNA and interrogated gene expression levels of ENG and PTPN14 in 111 paired samples of human primary pulmonary adenocarcinomas and matched uninvolved lung tissue from the same individual collected from lung cancer patients who had undergone debulking surgery for their cancer (Kim et al., 2013). We found that, in the uninvolved "normal" human lung tissue, which is angiogenically quiescent, PTPN14 gene expression levels were high but showed little variation across the panel ( $\cong 2$ fold) precluding robust eQTL analysis (Supplementary Figure S1). On the other hand, in the primary lung adenocarcinoma samples, although PTPN14 expression levels were lower than in the adjacent unaffected lung, they showed more variation between individuals across the panel ( $\cong 4$ fold $)$. Intriguingly, eQTL analysis showed association between the pulmonary AVM-associated ENG SNP, rs10987746-C, and higher expression of PTPN14 in this angiogenically-active adenocarcinoma tissue $\left(P=4 \times 10^{-3}\right.$, Figure 2), whereas $E N G$ transcript levels were not correlated with $r s 10987746$ in either normal or neoplastic lung tissue.

\section{EXPRESSION OF ENG, ACVRL1, AND PTPN14 ARE STRONGLY CORRELATED IN A GENETICALLY DIVERSE PANEL OF MOUSE LUNGS}

As PTPN14 gene expression levels were relatively homogeneous across the panel of normal angiogenically quiescent human lung tissues, we turned to a genetically heterogeneous mouse model to interrogate possible correlations between gene expression of the two HHT causative genes, Eng and Acvrll, with the pulmonary AVM modifier, Ptpn14. An F1 inter-specific mouse backcross between the two inbred mouse species, Mus spretus and Mus musculus FVB/Ola provides even greater genetic heterogeneity than seen within the human population, such that individual genes show a wider dynamic range of expression levels between individual mice, thus permitting more powerful expression correlation analysis (Quigley et al., 2009; Kim et al., 2013; Sjolund et al.,

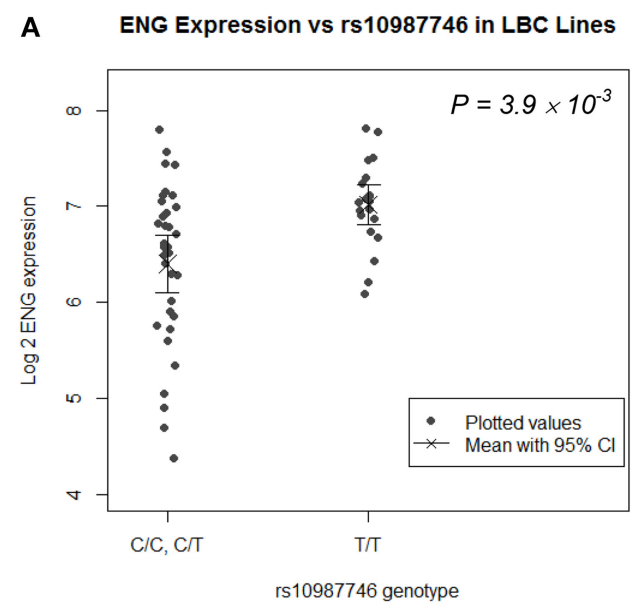

FIGURE 1 | ENG SNP rs10987746 correlates with ENG RNA levels in human LB cell lines. (A) ENG and (B) PTPN14 expression was assessed according to rs10987746 genotype in data derived from LB cell lines from Utah residents of Northern and Western European Ancestry from the CEPH collection (CEU) (Cheung et al., 2005). The
B PTPN14 Expression vs rs10987746 in LBC Lines

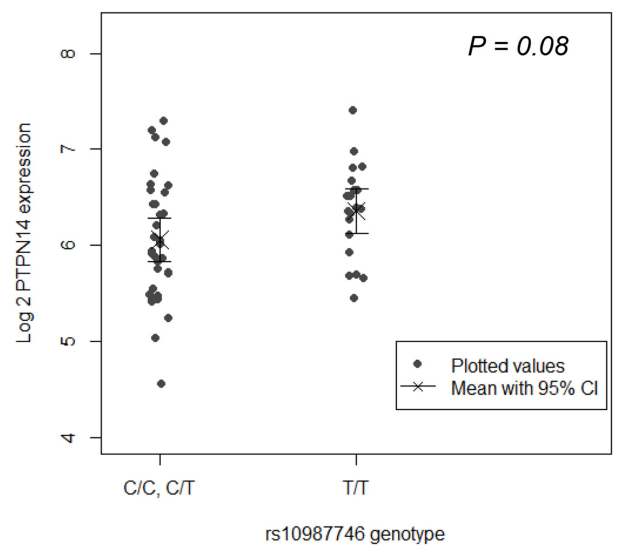

rs10987746-C/C genotype was unusually underrepresented in this panel $(n=7)$, and showed no statistically significant difference in expression from the rs10987746 -T/C genotype, hence these two genotypes were pooled. The mean gene expression and the $95 \%$ confidence interval of this mean are plotted for each genotype. 


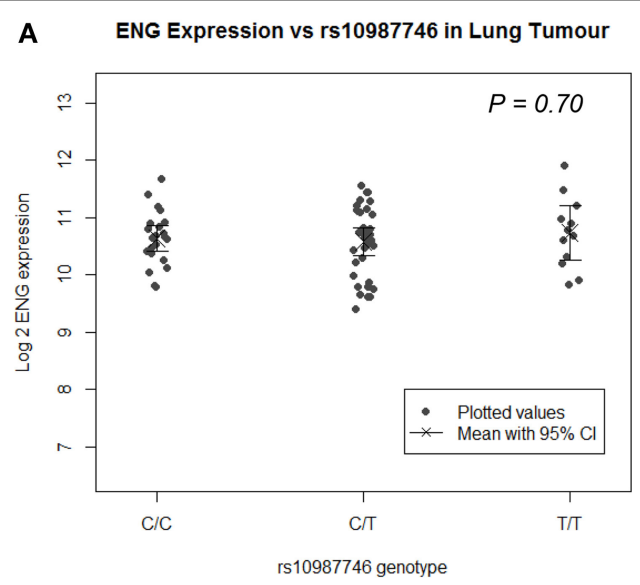

FIGURE 2 | ENG SNP rs 10987746 correlates with PTPN14 but not ENG expression levels in human primary pulmonary adenocarcinoma tissue, but not in normal lung. (A) ENG and (B) PTPN14 expression was plotted vs. rs 10987746 genotype in RNAs extracted from a panel of 111 primary human lung adenocarcinoma samples (A,B) and matched uninvolved lung tissue from

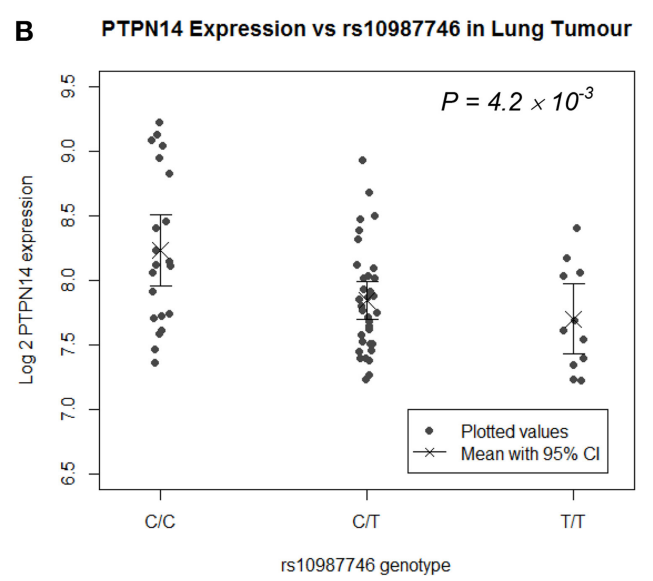

the same patients (insignificant see Supplementary Figure S1). The mean gene expression and the $95 \%$ confidence interval of this mean are shown for each genotype. Gene expression data was ascertained using Illumina human-6 v2.0 expression beadchip technology (Kim et al., 2013) (GEO GSE32665) and P-values (ANOVA) were calculated using Tukey's HSD in R.
2014). We quantified relative expression levels of Eng, Acvrl1, and Ptpn14 transcripts across this inter-specific normal mouse lung panel and found that the three test genes showed highly correlated expression levels with each other (Spearman's rho values from 0.75 to $0.9, P=1 \times 10^{-12}$; Figure 3 ). This suggests that Eng, Acvrl1, and Ptpn14 may all be expressed in the same lung cell type (Quigley et al., 2009; Kim et al., 2013), presumably endothelial, and that they are possibly co-regulated within that cell type (Sjolund et al., 2014). However, the data cannot exclude the possibility that tight expression correlation is due to crosstalk between two intimately interacting cell types.

\section{DISCUSSION}

The identification of modifier genes can be informative for elucidating possible molecular mechanisms regulating variable penetrance and expressivity of clinical symptoms of disease. In the current study we show that genetic variation in the wild type ENG allele, inherited from the unaffected parent, modifies HHT clinical outcome in terms of presence of pulmonary AVMs in HHT1. We found that the pulmonary AVM-at-risk allele, ENGrs10987746-C, associates with a small but significant reduction in $E N G$ transcript levels in a panel of human LB cell line data, and we demonstrate significant expression interaction between ENG, ACVRL1, and the pulmonary AVM modifier, PTPN14, by eQTL analysis and by expression correlation analysis of mouse and human lung tissue in vivo. We also report suggestive association between SNPs in EMILIN2, which encodes the extracellular matrix protein, elastin microfibril interfacer 2, in two independent cohorts of HHT1 patients from Netherlands and France.

There have been previous reports of genetic association between SNPs in ACVRL1 and ENG with sporadic brain AVMs in North American and Northern European Caucasians. Pawlikowska et al. (2005) demonstrated association of sporadic brain AVMs with ACVRL1-rs2071219, and marginal association with ENG-rs16930129 $(P=0.002)$ and ENG-rs10987759 $(P=$
0.09) in 177 sporadic brain AVM patients vs. 129 healthy controls. Simon et al. (2006) replicated the association between ACVRL1rs2071219 with brain AVMs or dural arteriovenous fistulas in a Northern European cohort, but were unable to show such association with ENG SNPs. Conversely, ACVRL1-rs2071219 association with sporadic brain AVMs was not reproduced in a more recent study of 143 Dutch Caucasian patients vs. 360 ethnically matched healthy volunteers (Boshuisen et al., 2013). Within HHT, Boshuisen et al. (2013) screened for genetic association between ENG SNPs and presence of brain AVMs within the Dutch HHT1 population by matched pair analysis of $24 \mathrm{HHT} 1$ patients with brain AVMs vs. 24 HHT1 relatives with no brain AVM, but they were unable to show genetic association, possibly due to small sample sizes of the brain AVM-positive HHT1 population. In the current study, we genotyped larger numbers of HHT1 patients and focused on pulmonary AVMs which is more common than brain AVM. We were able to screen for genetic association to the wild type ENG allele in 266 affected HHT1 individuals from 35 families, for whom we could deduce the phase of the wild type ENG SNP. We found weak but significant genetic association between the inheritance of a wild type ENG rs 10987746-C allele and increased risk of pulmonary AVMs in HHT1.

Here we complemented our previous HHT studies implicating PTPN14 as a genetic modifier of pulmonary AVMs by demonstrating strong expression correlation between Eng, Acvrl1, and Ptpn14 transcript levels in normal wild type mouse lung tissues. Our previous studies utilizing correlation gene expression network analysis of interspecific mouse backcrosses have demonstrated the power of this technique to define not only celltype-specific co-expression, but to identify genes whose protein products show direct physical or functional interactions within tissues (Quigley et al., 2009; Sjolund et al., 2014). The strong correlation between expression of these three genes, together with expression of other endothelial-specific genes (data not shown), suggests that in normal mouse lung Ptpn14, Eng, and Acvrl1 are 


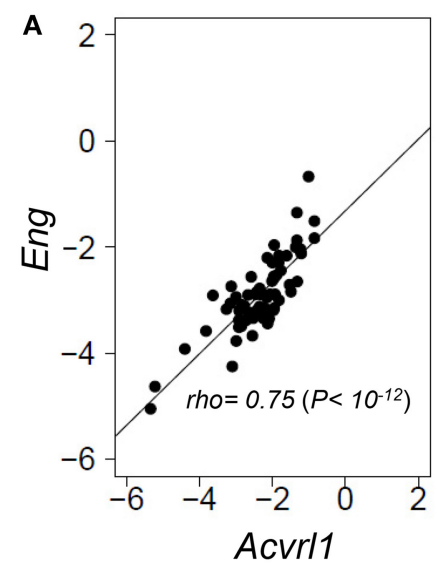

FIGURE 3 | Eng, Acvrl1, and Ptpn 14 gene expression are highly correlated in normal mouse lung tissues. RNA extracted from a panel of 69 wild type healthy mouse lungs from an F1 inter-specific mouse backcross between Mus spretus and Mus musculus FVB/Ola, was subjected to TAQMAN qRT-PCR analysis with five probes, Eng, Acvrl1, Ptpn14, Gus, and
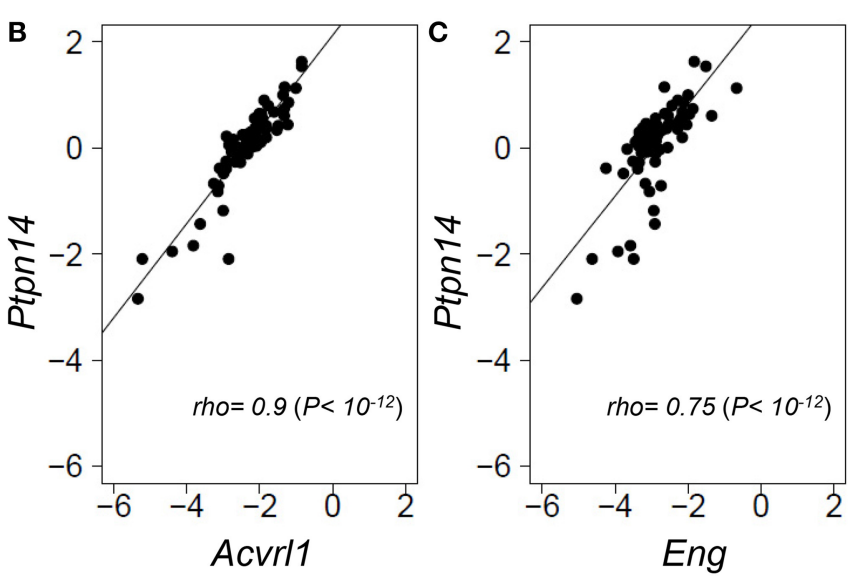

predominantly co-expressed within the same cell type, namely endothelial. However, there are currently no PTPN14-specific antibodies that perform well for immunohistochemical analysis to test this possibility.

We found that the pulmonary AVM-at-risk allele rs10987746$C$ associated with a slight but significantly lower level of ENG RNA in a human LB cell line panel, providing supporting evidence that genetic variation within this gene may influence its own expression. Conversely, in a panel of RNAs extracted from human primary lung adenocarcinomas and from adjacent normal lung tissue, there was no correlation between ENG rs10987746 and ENG expression level. This may, in part, be due to the technical limitations of working with primary human tissues compared to cell lines, and due to differences in the technological platforms used for gene expression analysis in the two experiments. However, in the human lung tumor tissues $r$ s10987746 did show correlation with expression levels of PTPN14, despite lack of association with $E N G$ transcript levels. A possible explanation is that this SNP is associated with differential post-transcriptional or translational control of endoglin levels that subsequently affect PTPN14 expression. rs10987746 lies within an antisense transcript showing $300 \mathrm{nt}$ complementarity to the ENG mRNA that might contribute to regulation of ENG in either cis or trans. Additionally, rs10987746 is proximal to the penultimate ENG exon which is subject to differential splicing to generate two structurally distinct and functionally antagonistic endoglin protein isoforms differing within their intracellular domains (Bellon et al., 1993; Lopez-Novoa and Bernabeu, 2010). Whether or not rs10987746 associates with differential ENG splicing, remains to be tested. Overall, however, we conclude that correlations between ENG, ALK1, and PTPN14 expression in both mouse and human lung tissues, support our previous in vitro studies of reciprocal regulation between $A C V R L 1$ and PTPN14 in primary human endothelial cells, and genetic association between PTPN14 and risk of pulmonary AVMs in HHT (Benzinou et al., 2012).
Gapdh. (A-C) show pair-wise correlations for expression of the two probes indicated in each of the 69 samples. Eng, Acvrl1, and Ptpn 14, expression are normalized against Gapdh. The data showed a similar strong correlation when normalized against Gus (not shown). Non-parametric Spearman Rank correlations shown were calculated in $\mathrm{R}$.
Since publishing our findings on PTPN14 involvement in HHT (Benzinou et al., 2012), several papers have demonstrated that, in epithelial cells, PTPN14 is a negative regulator of the transcriptional cofactors, Yap, and Taz (Huang et al., 2012; Liu et al., 2012; Wang et al., 2012; Wilson et al., 2014). These proteins are targets of the Hippo kinase pathway, originally identified as a master regulator of cell and organ size, mediator of contact inhibition and regulator of stem cell maintenance (Barry and Camargo, 2013; Hiemer and Varelas, 2013). More recently, Hippo signaling has been found to be a major regulator of mechanotransduction in both epithelial and endothelial cells (Dupont et al., 2011; Sansores-Garcia et al., 2011; Wada et al., 2011; Zhao et al., 2012; Aragona et al., 2013). Intriguingly, EMILIN2, which showed suggestive association with pulmonary AVMs and which is known to regulate angiogenesis, has also been reported to regulate Taz activity (Marastoni et al., 2014), suggesting some commonality in the downstream pathways of two pulmonary AVM modifier genes.

Blood vessels, in particular, are subject to constant shear stress and remodeling, suggesting that disruption of endothelial cell signaling pathways that regulate mechanotransduction within the vascular bed may contribute to defects in vascular remodeling and AVM susceptibility (Roman and Pekkan, 2012). The Roman group previously demonstrated an important link between the fish orthologue of $A C V R L 1$, alk1, and regulation of mechanotransduction when using the zebrafish as a model system. They demonstrated not only that alk 1 expression in arterial endothelial cells requires blood flow, but that under normal shear stress conditions a subset of flow-responsive genes are dysregulated in alk1 mutant arterial endothelial cells (Corti et al., 2011). They proposed a model whereby blood flow, under normal conditions, not only activates alk1 expression, but delivers cardiac-derived Bmp10 to activate an Alk1-responsive (flow-responsive) gene expression program that limits nascent arterial caliber (Laux et al., 2013). The interaction between PTPN14, endoglin, and ALK1 in mouse and human tissues provides another molecular link 
between the HHT causative genes and the mechanotransduction pathway. It will be important to elucidate the detailed molecular mechanisms regulating these interactions in endothelial cells, particularly within the lung, since this might lead to more rational choices for HHT therapy.

\section{AUTHOR CONTRIBUTIONS}

All authors made substantial contributions to the conception or design of the work or contributed to acquisition, analysis, or interpretation of data and assisted in drafting or revising the manuscript, and approved the final version and agree to be accountable for all aspects of the work, ensuring that questions related to the accuracy or integrity of any part of the work are appropriately investigated and resolved.

\section{ACKNOWLEDGMENTS}

Thanks to Minh Thu Luu, Mamie Higgins and Suprita Trilok for technical assistance. The primary SNP screen was undertaken by the UCSF Institute of Human Genetics Genome Core. Secondary SNP genotyping and RT-TAQMAN analysis of lung tissues was performed by the UCSF HDFCCC Genome Core. This work was funded by grants from the NIH (R01-HL078564 and R56HL122869) to RJA and from HHT International Foundation to RJA. MB. was the recipient of a fellowship from American Heart Association, and TGWL. was the recipient of a travel fellowship from the Ter Meulen Fund, NL.

\section{SUPPLEMENTARY MATERIAL}

The Supplementary Material for this article can be found online at: http://www.frontiersin.org/journal/10.3389/fgene.2015. 00067/abstract

\section{REFERENCES}

Akers, A. L., Johnson, E., Steinberg, G. K., Zabramski, J. M., and Marchuk, D. A. (2009). Biallelic somatic and germline mutations in cerebral cavernous malformations (CCMs): evidence for a two-hit mechanism of CCM pathogenesis. Hum. Mol. Genet. 18, 919-930. doi: 10.1093/hmg/ddn430

Aragona, M., Panciera, T., Manfrin, A., Giulitti, S., Michielin, F., Elvassore, N., et al. (2013). A mechanical checkpoint controls multicellular growth through YAP/TAZ regulation by actin-processing factors. Cell 154, 1047-1059. doi: 10.1016/j.cell.2013.07.042

Barry, E. R., and Camargo, F. D. (2013). The Hippo superhighway: signaling crossroads converging on the Hippo/Yap pathway in stem cells and development. Curr. Opin. Cell Biol. 25, 247-253. doi: 10.1016/j.ceb.2012.12.006

Bellon, T., Corbi, A., Lastres, P., Cales, C., Cebrian, M., Vera, S., et al. (1993). Identification and expression of two forms of the human transforming growth factor-beta-binding protein endoglin with distinct cytoplasmic regions. Eur. J. Immunol. 23, 2340-2345. doi: 10.1002/eji.1830230943

Benzinou, M., Clermont, F. F., Letteboer, T. G., Kim, J. H., Espejel, S., Harradine, K. A., et al. (2012). Mouse and human strategies identify PTPN14 as a modifier of angiogenesis and hereditary haemorrhagic telangiectasia. Nat. Commun. 3, 616. doi: $10.1038 /$ ncomms 1633

Boshuisen, K., Brundel, M., de Kovel, C. G., Letteboer, T. G., Rinkel, G. J., Westermann, C. J., et al. (2013). Polymorphisms in ACVRL1 and endoglin genes are not associated with sporadic and HHT-related brain AVMs in Dutch patients. Transl. Stroke Res. 4, 375-378. doi: 10.1007/s12975-012-0231-4

Cheung, V. G., Spielman, R. S., Ewens, K. G., Weber, T. M., Morley, M., and Burdick, J. T. (2005). Mapping determinants of human gene expression by regional and genome-wide association. Nature 437, 1365-1369. doi: 10.1038/nature04244

Corti, P., Young, S., Chen, C. Y., Patrick, M. J., Rochon, E. R., Pekkan, K., et al. (2011). Interaction between alk1 and blood flow in the development of arteriovenous malformations. Development 138, 1573-1582. doi: 10.1242/dev.060467
Cunha, S. I., and Pietras, K. (2011). ALK1 as an emerging target for antiangiogenic therapy of cancer. Blood 117, 6999-7006. doi: 10.1182/blood-2011-01-330142

Dupont, S., Morsut, L., Aragona, M., Enzo, E., Giulitti, S., Cordenonsi, M., et al. (2011). Role of YAP/TAZ in mechanotransduction. Nature 474, 179-183. doi: 10.1038 /nature 10137

Faughnan, M. E., Palda, V. A., Garcia-Tsao, G., Geisthoff, U. W., McDonald, J., Proctor, D. D., et al. (2011). International guidelines for the diagnosis and management of hereditary haemorrhagic telangiectasia. J. Med. Genet. 48, 73-87. doi: 10.1136/jmg.2009.069013

Garrido-Martin, E. M., Nguyen, H. L., Cunningham, T. A., Choe, S. W., Jiang, Z., Arthur, H. M., et al. (2014). Common and distinctive pathogenetic features of arteriovenous malformations in hereditary hemorrhagic telangiectasia 1 and hereditary hemorrhagic telangiectasia 2 animal models-brief report. Arterioscler. Thromb. Vasc. Biol. 34, 2232-2236. doi: 10.1161/ATVBAHA.114.303984

Guttmacher, A. E., Marchuk, D. A., and White, R. I. Jr. (1995). Hereditary hemorrhagic telangiectasia. N. Engl. J. Med. 333, 918-924. doi: 10.1056/NEJM199510053331407

Hiemer, S. E., and Varelas, X. (2013). Stem cell regulation by the Hippo pathway. Biochim. Biophys. Acta 1830, 2323-2334. doi: 10.1016/j.bbagen.2012. 07.005

Huang, J. M., Nagatomo, I., Suzuki, E., Mizuno, T., Kumagai, T., Berezov, A., et al. (2012). YAP modifies cancer cell sensitivity to EGFR and survivin inhibitors and is negatively regulated by the non-receptor type protein tyrosine phosphatase 14. Oncogene 32, 2220-2229. doi: 10.1038/onc.2012.231

Kawasaki, K., Freimuth, J., Meyer, D. S., Lee, M. M., Tochimoto-Okamoto, A., Benzinou, M., et al. (2014). Genetic variants of Adam17 differentially regulate TGFbeta signaling to modify vascular pathology in mice and humans. Proc. Natl. Acad. Sci. U.S.A. 111, 7723-7728. doi: 10.1073/pnas.1318761111

Kim, I. J., Quigley, D., To, M. D., Pham, P., Lin, K., Jo, B., et al. (2013). Rewiring of human lung cell lineage and mitotic networks in lung adenocarcinomas. Nat. Commun. 4, 1701. doi: 10.1038/ncomms2660

Lange, K., Cantor, R., Horvath, S., Perola, M., Sabatti, C., Sinsheimer, J., et al. (2001). Mendel version 4.0: a complete package for the exact genetic analysis of discrete traits in pedigree and population data sets. Am. J. Hum. Genet. 69, A1886 (Suppl.).

Lange, K., Sinsheimer, J. S., and Sobel, E. (2005). Association testing with Mendel. Genet. Epidemiol. 29, 36-50. doi: 10.1002/gepi.20073

Laux, D. W., Young, S., Donovan, J. P., Mansfield, C. J., Upton, P. D., and Roman, B. L. (2013). Circulating Bmp10 acts through endothelial Alk1 to mediate flow-dependent arterial quiescence. Development 140, 3403-3412. doi: 10.1242/dev.095307

Lebrin, F., Goumans, M. J., Jonker, L., Carvalho, R. L., Valdimarsdottir, G., Thorikay, M., et al. (2004). Endoglin promotes endothelial cell proliferation and TGF-beta/ALK1 signal transduction. EMBO J. 23, 4018-4028. doi: 10.1038/sj.emboj.7600386

Lesca, G., Olivieri, C., Burnichon, N., Pagella, F., Carette, M. F., Gilbert-Dussardier, B., et al. (2007). Genotype-phenotype correlations in hereditary hemorrhagic telangiectasia: data from the French-Italian HHT network. Genet. Med. 9, 14-22. doi: 10.1097/GIM.0b013e31802d8373

Letteboer, T. G., Mager, J. J., Snijder, R. J., Koeleman, B. P., Lindhout, D., Ploos van Amstel, J. K., et al. (2006). Genotype-phenotype relationship in hereditary haemorrhagic telangiectasia. J. Med. Genet. 43, 371-377. doi: 10.1136/jmg.2005.035451

Liu, X., Yang, N., Figel, S. A., Wilson, K. E., Morrison, C. D., Gelman, I. H., et al. (2012). PTPN14 interacts with and negatively regulates the oncogenic function of YAP. Oncogene 32, 1266-1273. doi: 10.1038/onc.2012.147

Lopez-Novoa, J. M., and Bernabeu, C. (2010). The physiological role of endoglin in the cardiovascular system. Am. J. Physiol. Heart Circ. Physiol. 299, H959-H974. doi: 10.1152/ajpheart.01251.2009

Marastoni, S., Andreuzzi, E., Paulitti, A., Colladel, R., Pellicani, R., Todaro, F., et al. (2014). EMILIN2 down-modulates the Wnt signalling pathway and suppresses breast cancer cell growth and migration. J. Pathol. 232, 391-404. doi: 10.1002/path.4316

McDonald, D. A., Shi, C., Shenkar, R., Gallione, C. J., Akers, A. L., Li, S., et al. (2014). Lesions from patients with sporadic cerebral cavernous malformations harbor somatic mutations in the CCM genes: evidence for a common biochemical pathway for CCM pathogenesis. Hum. Mol. Genet. 23, 4357-4370. doi: 10.1093/hmg/ddu153 
Pardali, E., Goumans, M. J., and Ten Dijke, P. (2010). Signaling by members of the TGF-beta family in vascular morphogenesis and disease. Trends Cell Biol. 20, 556-567. doi: 10.1016/j.tcb.2010.06.006

Park, S. O., Wankhede, M., Lee, Y. J., Choi, E. J., Fliess, N., Choe, S. W., et al. (2009). Real-time imaging of de novo arteriovenous malformation in a mouse model of hereditary hemorrhagic telangiectasia. J. Clin. Invest. 119, 3487-3496. doi: 10.1172/JCI39482

Pawlikowska, L., Tran, M. N., Achrol, A. S., Ha, C., Burchard, E., Choudhry, S., et al. (2005). Polymorphisms in transforming growth factor-beta-related genes ALK1 and ENG are associated with sporadic brain arteriovenous malformations. Stroke 36, 2278-2280. doi: 10.1161/01.STR.0000182253.91167.fa

Quigley, D. A., To, M. D., Perez-Losada, J., Pelorosso, F. G., Mao, J. H., Nagase, H., et al. (2009). Genetic architecture of mouse skin inflammation and tumour susceptibility. Nature 458, 505-508. doi: 10.1038/nature07683

R Development Core Team. (2011). R: A Language and Environment for Statistical Computing. Vienna: The R Foundation for Statistical Computing. Available online at: http://www.R-project.org/

Roman, B. L., and Pekkan, K. (2012). Mechanotransduction in embryonic vascular development. Biomech. Model. Mechanobiol. 11, 1149-1168. doi: 10.1007/s10237-012-0412-9

Sabba, C., Pasculli, G., Lenato, G. M., Suppressa, P., Lastella, P., Memeo, M., et al. (2007). Hereditary hemorrhagic telangiectasia: clinical features in ENG and ALK1 mutation carriers. J. Thromb. Haemost. 5, 1149-1157. doi: 10.1111/j.1538-7836.2007.02531.x

Sansores-Garcia, L., Bossuyt, W., Wada, K., Yonemura, S., Tao, C., Sasaki, H., et al. (2011). Modulating F-actin organization induces organ growth by affecting the Hippo pathway. EMBO J. 30, 2325-2335. doi: 10.1038/emboj.2011.157

Shovlin, C. L. (2010). Hereditary haemorrhagic telangiectasia: pathophysiology, diagnosis and treatment. Blood Rev. 24, 203-219. doi: 10.1016/j.blre.2010.07.001

Simon, M., Franke, D., Ludwig, M., Aliashkevich, A. F., Koster, G., Oldenburg, J., et al. (2006). Association of a polymorphism of the ACVRL1 gene with sporadic arteriovenous malformations of the central nervous system. J. Neurosurg. 104, 945-949. doi: 10.3171/jns.2006.104.6.945

Sjolund, J., Pelorosso, F. G., Quigley, D. A., Delrosario, R., and Balmain, A. (2014). Identification of Hipk2 as an essential regulator of white fat development. Proc. Natl. Acad. Sci. U.S.A. 111, 7373-7378. doi: 10.1073/pnas.1322275111

van Laake, L. W., van den Driesche, S., Post, S., Feijen, A., Jansen, M. A., Driessens, M. H., et al. (2006). Endoglin has a crucial role in blood cell-mediated vascular repair. Circulation 114, 2288-2297. doi: 10.1161/CIRCULATIONAHA.106.639161

Wada, K., Itoga, K., Okano, T., Yonemura, S., and Sasaki, H. (2011). Hippo pathway regulation by cell morphology and stress fibers. Development 138, 3907-3914. doi: 10.1242/dev.070987

Wang, W., Huang, J., Wang, X., Yuan, J., Li, X., Feng, L., et al. (2012). PTPN14 is required for the density-dependent control of YAP1. Genes Dev. 26, 1959-1971. doi: 10.1101/gad.192955.112

Wilson, K. E., Li, Y. W., Yang, N., Shen, H., Orillion, A. R., and Zhang, J. (2014) PTPN14 forms complex with Kibra and LATS1 proteins and negatively regulates the YAP oncogenic function. J. Biol. Chem. 289, 23693-23700. doi: 10.1074/jbc.M113.534701

Zhao, B., Li, L., Wang, L., Wang, C. Y., Yu, J., and Guan, K. L. (2012). Cell detachment activates the Hippo pathway via cytoskeleton reorganization to induce anoikis. Genes Dev. 26, 54-68. doi: 10.1101/gad.173435.111

Conflict of Interest Statement: The authors declare that the research was conducted in the absence of any commercial or financial relationships that could be construed as a potential conflict of interest.

Received: 29 November 2014; accepted: 09 February 2015; published online: 12 March 2015.

Citation: Letteboer TGW, Benzinou M, Merrick CB, Quigley DA, Zhau K, Kim I-J, To MD, Jablons DM, van Amstel JKP, Westermann CJJ, Giraud S, Dupuis-Girod S, Lesca G, Berg JH, Balmain A and Akhurst RJ (2015) Genetic variation in the functional ENG allele inherited from the non-affected parent associates with presence of pulmonary arteriovenous malformation in hereditary hemorrhagic telangiectasia 1 (HHT1) and may influence expression of PTPN14. Front. Genet. 6:67. doi: 10.3389/ fgene.2015.00067

This article was submitted to Genetic Disorders, a section of the journal Frontiers in Genetics.

Copyright (c) 2015 Letteboer, Benzinou, Merrick, Quigley, Zhau, Kim, To, Jablons, van Amstel, Westermann, Giraud, Dupuis-Girod, Lesca, Berg, Balmain and Akhurst. This is an open-access article distributed under the terms of the Creative Commons Attribution License (CC BY). The use, distribution or reproduction in other forums is permitted, provided the original author(s) or licensor are credited and that the original publication in this journal is cited, in accordance with accepted academic practice. No use, distribution or reproduction is permitted which does not comply with these terms. 